\title{
Neutrino signals from electroweak bremsstrahlung in solar WIMP annihilation
}

\author{
Nicole F. Bell, ${ }^{a}$ Amelia J. Brennan ${ }^{a}$ and Thomas D. Jacques ${ }^{a, b}$ \\ ${ }^{a}$ ARC Centre of Excellence for Particle Physics at the Terascale \\ School of Physics, The University of Melbourne, Victoria 3010, Australia \\ ${ }^{b}$ Department of Physics and School of Earth and Space Exploration, Arizona State Univer- \\ sity, Tempe, AZ 85287-1404, USA \\ E-mail: n.bell@unimelb.edu.au,a.brennan@pgrad.unimelb.edu.au, \\ thomas.jacques@asu.edu
}

\begin{abstract}
Bremsstrahlung of $W$ and $Z$ gauge bosons, or photons, can be an important dark matter annihilation channel. In many popular models in which the annihilation to a pair of light fermions is helicity suppressed, these bremsstrahlung processes can lift the suppression and thus become the dominant annihilation channels. The resulting dark matter annihilation products contain a large, energetic, neutrino component. We consider solar WIMP annihilation in the case where electroweak bremsstrahlung dominates, and calculate the resulting neutrino spectra. The flux consists of primary neutrinos produced in processes such as $\chi \chi \rightarrow \bar{\nu} \nu Z$ and $\chi \chi \rightarrow \bar{\nu} \ell W$, and secondary neutrinos produced via the decays of gauge bosons and charged leptons. After dealing with the neutrino propagation and flavour evolution in the Sun, we consider the prospects for detection in neutrino experiments on Earth. We compare our signal with that for annihilation to $W^{+} W^{-}$, and show that, for a given annihilation rate, the bremsstrahlung annihilation channel produces a larger signal by a factor of a few.
\end{abstract}




\section{Contents}

1 Introduction $\quad 1$

2 Neutrino production $\quad 2$

2.1 Dark matter coupling 3

2.2 Neutrino spectra at production 3

2.2.1 Primary neutrino spectra 4

2.2.2 Secondary neutrino spectra 5

3 Propagation in the Sun $\quad 6$

$\begin{array}{lll}3.1 & \text { Scattering and absorption } & 7\end{array}$

3.2 Flavour oscillations $\quad 8$

$\begin{array}{lll}3.3 & \text { Final spectra } & 9\end{array}$

4 Signals at Earth $\quad 11$

4.1 Muon event rates 11

$\begin{array}{lll}4.2 & \text { Parameter constraints } & 13\end{array}$

5 Conclusions

\section{Introduction}

High-energy neutrinos produced by dark matter (DM) self-annihilation in the Sun offer an appealing signal for indirect DM detection [1-8]. See, e.g., refs. [9-15] for recent work on this topic. DM particles which pass through the Sun can scatter, lose energy, and become captured by the gravitational field of the Sun. They accumulate in the Sun's core and eventually reach a density where self-annihilation becomes important. Most Standard Model (SM) particles produced in the annihilations interact in the Sun and are hence absorbed, while neutrinos will escape to be detected in experiments on Earth. The neutrinos may be produced directly in the DM annihilations, or as secondaries in the decays of other SM particles. A high energy neutrino signal from the Sun would thus be a compelling indication of dark matter.

The strength of this signal is controlled by the DM-nucleon scattering cross section which controls the DM capture rate and, if in equilibrium, the annihilation rate. Existing limits have been placed by Super-Kamiokande [16], IceCube [17-19], and other experiments. The strength of these limits depends on the annihilation channel assumed for dark matter, with limits for soft annihilation channels such as $\chi \chi \rightarrow \bar{b} b$ being weaker than those for hard channels such as $\chi \chi \rightarrow W^{+} W^{-}$. A comprehensive study of the neutrino spectra for the main annihilation channels with 2-body final states was performed in ref. [9]. In the present article we study DM annihilation processes with 3-body final states, namely bremsstrahlung processes in which a $W$ or $Z[20,21]$ or $\gamma[22-27]$ is radiated. In certain models, these bremsstrahlung processes have been shown to be the dominant DM annihilation mode.

For non-relativistic dark matter, it is useful to parametrise the annihilation cross section as

$$
\sigma_{A} v=a+b v^{2}+\mathcal{O}\left(v^{4}\right)
$$


where $v$ is the dark matter relative velocity (in units of $c$ ). The $a$ term arises only from $s$-wave annihilation, and the $L^{t h}$ partial wave contribution is suppressed by a factor $v^{2 L}$. For highly non-relativistic DM with $v \ll 1, s$-wave annihilations, if permitted, are thus expected to dominate. In the galactic halo, $v^{2} \sim 10^{-6} ; v$ is even smaller for the DM accumulated in the solar core. However, in many Majorana DM models, $s$-wave annihilation to a pair of fermions, $\chi \chi \rightarrow f \bar{f}$, is helicity suppressed by a factor of $\left(m_{f} / M_{\chi}\right)^{2}$ due to a mismatch between the net helicity and chirality of the fermion pair [28, 29]. A well known example is the annihilation of neutralino dark matter in supersymmetric models.

The circumstances in which annihilation to a pair of fermions is velocity and/or helicity suppressed were extensively detailed in ref. [21]. It is well known that radiation of a photon is able to lift this suppression via the electromagnetic (EM) bremsstrahlung process $\chi \chi \rightarrow$ $\bar{f} f \gamma[22-27]$. However, as shown in refs. [20, 21], electroweak (EW) bremsstrahlung is also able to lift this helicity suppression, such that the $\chi \chi \rightarrow \bar{f} f Z, \chi \chi \rightarrow f \nu W$, and $\chi \chi \rightarrow \bar{f} f \gamma$ annihilation channels all dominate over $\chi \chi \rightarrow \bar{f} f$. Physically, the suppression is lifted because the emitted gauge boson carries away a unit of angular momentum, allowing a fermion spinflip, so there is no longer a mismatch between the chirality of the leptons and their allowed 2-particle spin state. Relative to the 2-body process, the rate for the 3 -body final state suffers a suppression factor from the radiating particle given by $\alpha / \pi$. However, this may be more than compensated for by the consequent removal of the $\left(m_{f} / M_{\chi}\right)^{2}$ suppression factor.

The importance of these radiative corrections to the dark matter annihilation process have been examined in a number of recent papers [30-41], with a focus on the indirect detection of dark matter due to annihilations in the Galactic halo. Interestingly, a possible hint of an EM bremsstrahlung signal was found in recent Fermi data [42]. In the present paper we consider the indirect detection of dark matter via annihilation in the Sun, noting that the EW bremsstrahlung annihilation channels produce large, energetic, neutrino fluxes.

This paper aims to produce the final neutrino energy spectra, as they would be detected on Earth, for solar WIMP annihilation via the EW bremsstrahlung process, and to compare the resulting signal with that for other commonly assumed annihilation channels. In section 2 we describe the neutrino spectra at production, including primary neutrinos produced directly in the annihilations, and secondaries produced from the decay of emitted gauge bosons. In section 3 we consider the scattering and flavour evolution of the neutrinos as they propagate through the Sun, to determine the final spectra, while in section 4 we compare the event rates for bremsstrahlung annihilation with those for other DM annihilation modes. Our results are summarised briefly in section 5 .

\section{Neutrino production}

During the process of capture in the Sun, DM particles scatter elastically with solar nuclei, losing enough energy to become gravitationally bound. The capture rate, $C^{\odot}$, is proportional to the DM/solar nucleon scattering cross section and depends upon astrophysical parameters such as the local DM velocity and density. Once captured, subsequent scattering causes the $\mathrm{DM}$ to become concentrated in the solar core, in a region of size $\sim 0.01 R_{\odot} \sqrt{100 \mathrm{GeV} / M_{\chi}}$, where $R_{\odot}$ is the solar radius; the region is sufficiently small that we may assume all DM annihilations occur in the centre of the Sun [9].

The differential neutrino flux is given by

$$
\frac{d N_{\nu}}{d E}=\frac{\Gamma_{\text {ann }}}{4 \pi d^{2}} \sum_{k} \mathrm{BR}_{k} \frac{d N_{k}}{d E}
$$


where $d$ is the distance from the centre of the Sun to the detector, and $B R_{k}$ is the branching ratio to a specific final state, $k$. The annihilation rate $\Gamma_{\text {ann }}$ depends on the capture rate $C^{\odot}$ as

$$
\Gamma_{\text {ann }}=\frac{1}{2} C^{\odot} \tanh ^{2}\left(t_{0} / \tau_{A}\right),
$$

where $t_{0}=4.5 \mathrm{Gyr}$ is the age of the Sun and $\tau_{A}$ is the time-scale for competing capture and annihilation processes $[6,9,43-45]$. This time scale is given by $\tau_{A}=1 / \sqrt{C^{\odot} A^{\odot}}$, where $A^{\odot}=\langle\sigma v\rangle / V_{\text {eff }}$ is the annihilation cross section times the relative WIMP velocity per volume (see, e.g. the reviews in $[46,47]$ for explicit expressions for these rates). This time-scale is often much smaller than $t_{0}$, such that an equilibrium is reached between the capture and annihilation processes with $\Gamma_{\text {ann }} \simeq C^{\odot} / 2$. In this case, it is the DM-nucleon scattering cross section, rather than the annihilation cross section, which determines the annihilation rate. We shall return to the question of capture-annihilation equilibrium in section 4.2.

\subsection{Dark matter coupling}

We use the leptophilic Majorana DM coupling of ref. [48] as a simple example of a scenario in which EW bremsstrahlung dominates the annihilation. The DM is a gauge-singlet Majorana fermion which annihilates to leptons via the interaction term

$$
f\left(\nu l^{-}\right)_{L} \varepsilon\left(\begin{array}{c}
\eta^{+} \\
\eta^{0}
\end{array}\right) \chi+h . c .=f\left(\nu_{L} \eta^{0}-l_{L} \eta^{+}\right) \chi+\text { h.c. }
$$

where $f$ is a coupling constant, $\varepsilon$ is the $2 \times 2 \mathrm{SU}(2)$ invariant antisymmetric matrix, and $\left(\eta^{+}, \eta^{0}\right)$ form a new $S U(2)$ doublet scalar which mediates the annihilation. An identical coupling arises in supersymmetric models if we take $\chi$ to be a (bino-like) neutralino and $\eta$ a sfermion doublet.

Ref. [48] proposed this model as an explanation of the sharp excess in the cosmic ray $e^{+} /\left(e^{-}+e^{+}\right)$fraction at energies beyond $\sim 10 \mathrm{GeV}$, as recently measured by the PAMELA experiment [49-51]. Here we adopt this model as a simple prototype of the situation in which bremsstrahlung processes dominate the DM annihilation. As explained in [20,21], the $s$-wave contributions to the lowest order annihilation processes $\chi \chi \rightarrow \bar{\nu} \nu, e^{+} e^{-}$are suppressed by factors of $\left(m_{l} / M_{\chi}\right)^{2}$. Bremsstrahlung lifts this suppression to become the dominant annihilation process.

We shall consider three possibilities for flavour structure of the branching ratios: DM annihilates to all three lepton flavours equally $\left(f_{e}: f_{\mu}: f_{\tau}=1: 1: 1\right)$, to electron-flavoured leptons only $\left(f_{e}: f_{\mu}: f_{\tau}=1: 0: 0\right)$, or to $\mu$ and $\tau$ flavours equally $\left(f_{e}: f_{\mu}: f_{\tau}=0: 1: 1\right)$. However, we shall see that neutrino mixing reduces the sensitivity of the final results to the flavour structure of the couplings.

\section{$2.2 \quad$ Neutrino spectra at production}

We determine the energy spectra for the neutrinos at production by adding the contributions from the primary neutrinos produced directly in DM annihilation, and the secondary neutrino produced by decays of gauge bosons or charged leptons. Throughout, we neglect neutrinos with energies less than $\sim 0.5 \mathrm{GeV}$. Note that IceCube, for example, has a detection threshold of $\sim 100 \mathrm{GeV}$ [52], which is reduced to $\sim 10 \mathrm{GeV}$ with the addition of DeepCore [53]. We shall be particularly interested in the higher-energy regions of the spectra, as the bremsstrahlung process leads to neutrino spectra with a distinct high energy peak; moreover, detection cross sections grow (approximately linearly) with energy. 


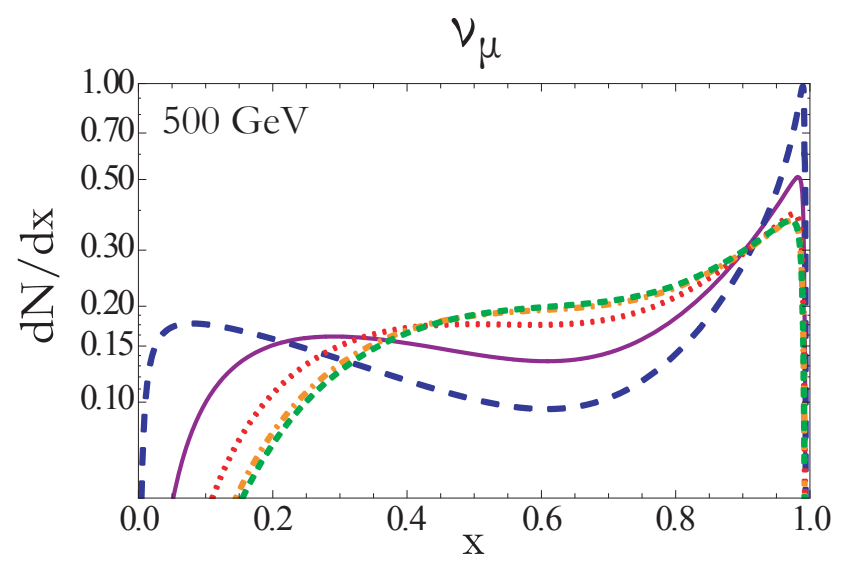

Figure 1. The primary spectra of muon-flavoured neutrinos for $\mu=M_{\eta}^{2} / M_{\chi}^{2}=1.01$ (dashed, blue), $\mu=1.2$ (solid, purple), $\mu=2$ (red, dotted), $\mu=5$ (orange, dot-dashed) and $\mu=10$ (green, smalldashed). Increasing $\mu$ reduces the high-energy peak but increases the number of mid-range energy neutrinos. We have taken the annihilations to have equal branching ratios to all lepton flavours, $f_{e}=f_{\mu}=f_{\tau}$. Note that the $x$-axis is defined as $x \equiv E_{\nu} / M_{\chi}$.

\subsubsection{Primary neutrino spectra}

We first consider the primary neutrinos, those produced from the direct DM annihilation channels:

$$
\begin{aligned}
& \chi \chi \rightarrow l^{-} \nu_{l} W^{+}, \\
& \chi \chi \rightarrow l^{+} \bar{\nu}_{l} W^{-}, \\
& \chi \chi \rightarrow \nu_{l} \bar{\nu}_{l} Z .
\end{aligned}
$$

The spectra of primary neutrinos from these processes are given in [20], along with calculations for the relative cross sections. The contribution from each bremsstrahlung channel is included according to these relative cross sections; for example the branching ratio for emission of a $W^{-}$is given by $\sigma_{e \nu W} / \sigma_{\text {total }}$, where $\sigma_{\text {total }}$ is found by adding all the bremsstrahlung components together (including the contributions from $\chi \chi \rightarrow l^{+} l^{-} Z$ and $\chi \chi \rightarrow l^{+} l^{-} \gamma$, which do not produce primary neutrinos). Annihilation directly to neutrinos via the suppressed process $\chi \chi \rightarrow \nu \bar{\nu}$ would produce a monoenergetic spectrum with $E_{\nu}=M_{\chi}$; we neglect the contribution of this subdominant process in our analysis.

The primary neutrino spectrum is sensitive to the ratio of the DM and propagator masses, $\mu=\frac{M_{\eta}^{2}}{M_{\chi}^{2}}$. We focus on small values of $\mu$ as this maximises the branching ratio of the bremsstrahlung processes relative to the 2-body final state annihilation processes; however, bremsstrahlung remains the dominant annihilation channel for $\mu \lesssim 10$. In figure 1 we plot the primary neutrino spectra for various choices of $\mu$. The spectra have a broad energy distribution, and a distinctive high energy peak at $x \simeq 1$. Increasing $\mu$ decreases the height of this peak, but increases the number of neutrinos of mid-range energy. For the remainder of this paper we shall assume a value of $\mu=1.2$ (but will also show results for $\mu=5$ in table $1)$.

In figure 2(a) the primary spectra of muon neutrinos are indicated by the dashed green line. Here we assume equal branching to all lepton flavours, and so the primary spectra are flavour-independent; changing the branching ratios will clearly affect the flavour dependence. Note that the primary neutrinos are the dominant contribution to the total neutrino spectrum 

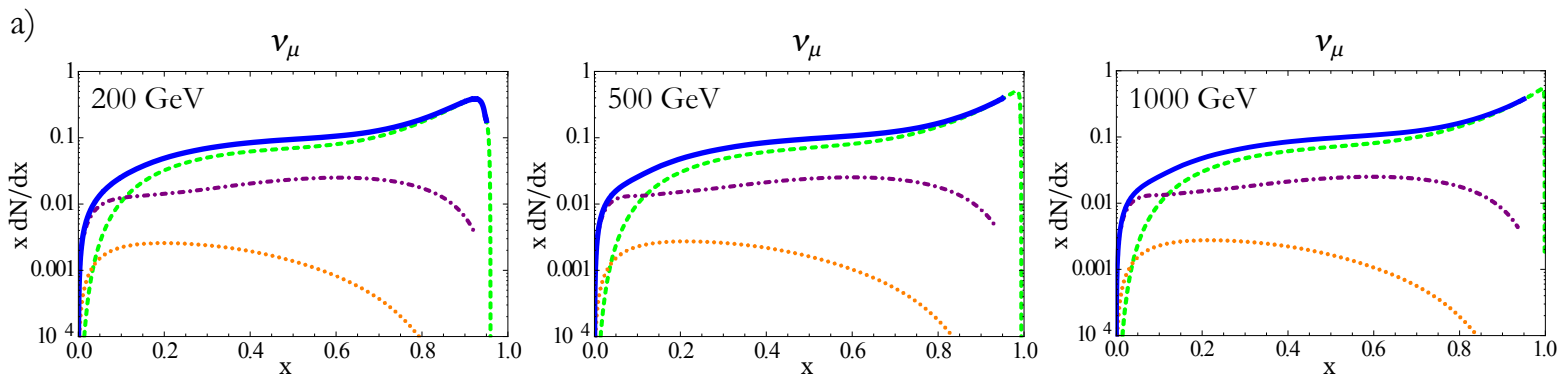

b)
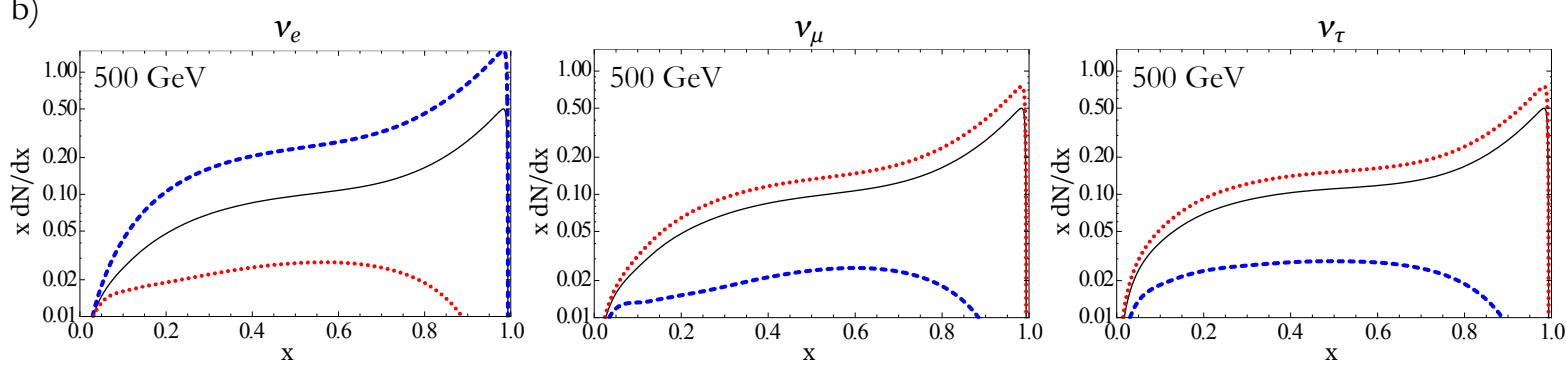

Figure 2. a) The contributions to the injected spectra, assuming branching to all lepton flavours equally, from primary neutrinos (green, dashed), secondary neutrinos from gauge boson decay (purple, dot-dashed) and secondary neutrinos from decay of primary taus (orange, dotted); the solid blue line shows the total. b) The total injected spectra for different branching ratios: annihilation to all lepton flavours equally (black, solid), annihilation to $e$-flavoured leptons only (blue, dashed), and annihilation to $\mu$ and $\tau$ flavours equally (red, dotted). All anti-neutrino spectra are identical to the corresponding neutrino spectra, and so are not shown. Note that the $x$-axis is defined as $x \equiv E_{\nu} / M_{\chi}$, and that we are using $\mu=1.2$.

for each flavour (shown as the solid blue line in figure 2(a)), particularly for the high-energy region of interest. Comparing the spectra for different choices of the DM mass, we see that the primary spectra maintain the same general shape. However, increasing the DM mass relative to $M_{W / Z}$ decreases the effect of kinematic thresholds and moves the peak closer to $x=1$. The antineutrino spectra are identical to the corresponding neutrino spectra.

\subsubsection{Secondary neutrino spectra}

We next consider the secondary neutrinos that are produced from gauge boson decays, or the decays of primary tau leptons. The decay of the emitted gauge boson is modelled using the Monte Carlo simulator PYTHIA [54], in which we create a $W$ or $Z$ at rest which is then allowed to decay with constraints to be outlined below. The neutrino spectra from the gauge boson decays are then boosted into the lab frame as per the appendix of ref. [21]. In the Sun, the core density is $\rho=140 \mathrm{~g} \mathrm{~cm}^{-3}$, so energy loss processes are relatively important as the DM annihilation products may interact with solar material and lose energy before decaying further. Adopting a procedure similar to that in ref. [9], the relevant energy losses are accounted for as described below.

Gauge bosons: Once produced, the lifetimes of the $W$ and $Z$ bosons are short enough that they decay before interacting and losing any significant amount of energy. Therefore, in PYTHIA, we set these bosons to decay as if in vacuum. The products of the hadronic decay modes of the gauge bosons are dealt with as explained below.

Charged leptons: The typical stopping time of a charged lepton is $\tau_{\mathrm{stop}} \sim 10^{-11} \mathrm{~s}$. If this is shorter than the boosted lifetime, $\tau_{\text {stop }}<\gamma \tau_{\text {dec }}$, the lepton will lose all its energy before decaying. For the energy range of interest, $E \lesssim 1 \mathrm{TeV}$, the muons are stopped before they 
decay while taus decay without losing a significant amount of energy. Thus, in the PYTHIA routine, we discard electrons and muons, and allow taus to decay as if in vacuum.

Quarks: Heavy hadrons have comparable $\tau_{\text {stop }}$ and $\gamma \tau_{\text {dec }}$, of order $10^{-11} \mathrm{~s}$ and $10^{-12} \mathrm{~s}$ respectively, so will often lose some energy before decaying; light hadrons are usually stopped before decay. As a reasonable, simple, approximation, we allow heavy hadrons (those including charm, top and bottom quarks) to decay, while light hadrons are discarded.

The resulting neutrino spectra from $W^{ \pm}$and $Z$ decay, as seen in the lab frame, are shown by the dot-dashed purple line in figure 2(a). These secondary neutrinos pile up and become dominant at low energies, as they are often produced through a chain of decays starting at the gauge boson. For this case of even branching ratios $\left(f_{e}=f_{\mu}=f_{\tau}\right)$ the secondary spectra of $\nu_{e}$ and $\nu_{\mu}$ are equal, as light hadrons and muons are stopped before decay, while $\tau, c$ and $b$ produce equal numbers of each. As with the primary neutrinos, the antineutrino spectra are identical to their corresponding neutrino spectra.

In the case where the coupling $f_{\tau}$ is non-zero, there will be a primary tau component which will decay to contribute to the secondary neutrino flux. As with the gauge bosons decays, the secondary neutrino spectra from tau decay (again created using PYTHIA) must be boosted into the lab frame and convolved with the primary tau spectrum. The neutrino spectra resulting from this process are shown by the dotted orange line in figure 2(a). The tau decays make a minor contribution to the $\nu_{e}$ and $\nu_{\mu}$ fluxes and a larger, though still subdominant, contribution to the $\nu_{\tau}$ flux.

Adding the contributions of primary and secondary neutrino fluxes, we plot the total spectra at production as the solid blue line in figure 2(a). The total spectra are also shown in figure 2(b) for varied branching ratios. We note that the primary neutrinos make the dominant contribution to the total neutrino flux (summed over all flavours), while for particular choices of the flavour branching ratios the secondary neutrinos can dominate the flux for an individual flavour. However, once neutrino mixing is taken into account the primary neutrinos will make the dominant contribution to the flux for each flavour. Therefore, our results are robust against uncertainties in the calculation of the secondary neutrinos, and instead are determined mainly by the flux of primary neutrinos (whose spectra can be calculated analytically).

\section{Propagation in the Sun}

Following production, the neutrino flux is modified by flavour oscillations, and by scattering with solar nuclei, which results in some absorption of the flux at high energy. These effects are well-understood, and various techniques exist to deal with them; we shall follow the method of ref. [55] to account for the scattering interaction, and then adopt results of ref. [56] when evaluating the flavour evolution. Many authors combine scattering and mixing effects into a single $3 \times 3$ density matrix equation that describes the neutrino evolution. However in the energy range of interest, $E_{\nu} \lesssim 1 \mathrm{TeV}$, oscillations and scattering approximately decouple [55], as the matter effects in the Sun ensure negligible mixing between $\nu_{e}$ and $\nu_{\mu, \tau}$ until after scattering becomes unimportant. We assume throughout that the $\nu_{\mu}$ and $\nu_{\tau}$ fluxes are equal, as $\nu_{\mu}$ and $\nu_{\tau}$ mix maximally (to good approximation) and, for the energy range of interest, the oscillation length is less than or approximately equal to the interaction length [55]. 


\subsection{Scattering and absorption}

The evolution equation, used to evolve the neutrino flux from production to $r_{c}$ (the radius where interactions become unimportant), has the form

$$
\frac{\partial \rho_{l}}{\partial r}=\left.\frac{\partial \rho_{l}}{\partial r}\right|_{\mathrm{inj}}+\left.\frac{\partial \rho_{l}}{\partial r}\right|_{\mathrm{NC}}+\left.\frac{\partial \rho_{l}}{\partial r}\right|_{\mathrm{CC}},
$$

for neutrinos of flavour $l$, where $\rho(r, E) d E$ is the neutrino flux. The first term on the right corresponds to the neutrino injection due to the annihilation of DM (including all secondary neutrinos). The second and third terms correspond to neutrino scattering through neutral current and charged current interactions respectively.

The probability of an interaction between a neutrino and a solar nucleon is given by

$$
P_{\text {int }}=\int_{0}^{R_{\odot}} \sigma_{\text {tot }}(E) N_{S}\left(r^{\prime}\right) d r^{\prime}=\frac{E}{\mathcal{E}},
$$

where $\sigma_{\text {tot }}$ is the neutrino total interaction cross section and $N_{S}(r) \simeq N_{0} e^{-r / \kappa R_{\odot}}$ is the nucleon density of the Sun, with $\kappa=0.1$ and $N_{0}=1.3 \times 10^{26} \mathrm{~cm}^{-3}$. The constants $\mathcal{E}$ parametrise the energy scale at which interactions become significant, and are given by

$$
\mathcal{E}_{\nu} \simeq 140 \mathrm{GeV}, \quad \mathcal{E}_{\bar{\nu}} \simeq 213 \mathrm{GeV} .
$$

Note that the total interaction cross section combines neutral current (NC) scatterings $\nu_{l} N \leftrightarrow \nu_{l} N$ and $\bar{\nu}_{l} N \leftrightarrow \bar{\nu}_{l} N$ (effectively removing a neutrino from the flux and re-injecting it at lower energy), and charged current (CC) scatterings $\nu_{l} N \leftrightarrow l^{-} N^{\prime}$ and $\bar{\nu}_{l} N \leftrightarrow l^{+} N^{\prime}$ (effectively removing a neutrino from the flux and producing an almost collinear charged lepton). If $l=e, \mu$ then the final lepton loses most, if not all, of its energy before it can decay again, and so the neutrino is considered absorbed. If $l=\tau$, the resulting $\tau^{ \pm}$decays promptly, either leptonically or hadronically.

Defining the optical depth as

$$
y(r) \equiv \frac{1}{N_{0} \kappa R_{\odot}} \int_{0}^{r} N_{S}\left(r^{\prime}\right) d r^{\prime}
$$

the evolution equation (3.1) takes the form

$$
\frac{\partial \rho_{j}}{\partial y}=h(E)+\frac{1}{\mathcal{E}}\left[-E \rho_{j}(y, E)+\int_{E}^{\infty} d E^{\prime} g_{j}\left(E / E^{\prime}\right) \rho_{j}(y, E)\right] \text {. }
$$

The term $h(E)$ represents our injected flux, the second term accounts for absorption of the flux through scattering, and the last term the reinjection or regeneration of neutrinos at lower energy. At high energies, absorption through scattering is a significant effect. Tau regeneration is also important, as absorbed neutrinos are repopulated and pile-up at lower energies. Again, this effect is most significant for large DM mass. The regeneration functions $g_{i}$ are given, e.g., in refs. [9, 55]. Following ref. [55] we use a perturbative solution of eq. (3.5) to handle the regeneration. For simplicity, we take $g_{i}=0.5$ and have checked that for the largest $M_{\chi}$ we consider (where regeneration is expected to occur most strongly) this simplification produces an error $<20 \%$ in the final spectra for energies $\gtrsim 100 \mathrm{GeV}$, which does not qualitatively change our conclusions. 


\subsection{Flavour oscillations}

We now include the effect of neutrino flavour mixing. We use the neutrino mixing parameters given in ref. [57], and assume the normal mass hierarchy, where $m_{1}<m_{2}<m_{3}$. We shall adopt a non-zero value for $\theta_{13}$, as indicated by recents results from the T2K [58] and the Daya Bay [59] collaborations, and demonstrate how the results change as we vary the value of this parameter.

Neutrinos undergo flavour conversion during their propagation through the Sun. These flavour effects differ from oscillations in vacuum due to the presence of neutrino refractive indices. In the dense solar medium, the electron neutrinos experience a matter potential of $V_{e}(r)=\sqrt{2} G_{F} N_{e}(r)$, where $N_{e}$ is the electron number density. At high density, this leads to an approximate alignment between the flavour and effective mass eigenstates, and suppresses oscillations of $\nu_{e}$ with $\nu_{\mu, \tau}$. The matter potential decreases as the neutrinos propagate outward from the centre of the Sun, and the neutrinos undergo an MSW resonance [60, 61] when

$$
\Delta m^{2} / 2 E \simeq V_{e}
$$

At low energies, the propagation through this resonance is adiabatic and the neutrinos remain in given mass eigenstates. At higher energies the evolution becomes non-adiabatic, with the neutrinos experiencing a level-crossing, or jump between mass eigenstates. Assuming an exponentially varying solar profile $\left(\rho(r) \propto e^{-r / r_{0}}\right)$, with $r_{0}=R_{\odot} / 10.54$, the crossing probability is conventionally given by $[62]$

$$
P_{C}=\frac{e^{\gamma \cos ^{2} \theta}-1}{e^{\gamma}-1}, \gamma=4 \pi r_{0} \Delta
$$

where $\Delta \equiv \Delta m^{2} / 4 E_{\nu}$. For antineutrinos, the $\bar{\nu}_{e}$ potential is $-V_{e}(r)$, which is equivalent to making the replacement $\theta \rightarrow \frac{\pi}{2}-\theta[63]$, thus

$$
\bar{P}_{C}=P_{C}\left(\theta \rightarrow \frac{\pi}{2}-\theta\right)
$$

We shall be concerned with two level crossings, controlled by $\Delta m_{21}^{2}$ and $\Delta m_{32}^{2}$ respectively. As $\Delta m_{32}^{2}$ is almost two orders of magnitude larger than $\Delta m_{21}^{2}$ the crossings decouple and can be treated independently. This separation is useful as it allows us to use a simple two-neutrino formalism which we apply to each crossing independently. This leads to

$$
P=\left(\begin{array}{ccc}
1-P_{C}^{l} & P_{C}^{l} & 0 \\
P_{C}^{l} & 1-P_{C}^{l} & 0 \\
0 & 0 & 1
\end{array}\right)\left(\begin{array}{ccc}
1 & 0 & 0 \\
0 & 1-P_{C}^{h} & P_{C}^{h} \\
0 & P_{C}^{h} & 1-P_{C}^{h}
\end{array}\right),
$$

where $P_{j k}$ represents the probability of a neutrino that originated in matter mass eigenstate $k$ ending up in vacuum mass eigenstate $j$. Here, $P_{C}^{l}$ and $P_{C}^{h}$ correspond to the lower and higher level crossing probabilities, driven by $\theta_{12}$ and $\theta_{13}$ respectively. See ref. [64] for a detailed discussion.

We shall need to evaluate the level crossing probabilities for the two resonances, and for both neutrinos and antineutrinos. In some of these cases the resonance condition (3.6) is never actually met; nonetheless, the level crossing probability is non-zero and the expression given in eq.(3.7) still holds [56, 65, 66]. ${ }^{1}$ For some parameters of interest (namely, high

\footnotetext{
${ }^{1}$ As discussed in refs. $[56,66]$, the crossing probability is largest when adiabaticity is maximally violated, and is non-zero even away from resonance.
} 
(a)

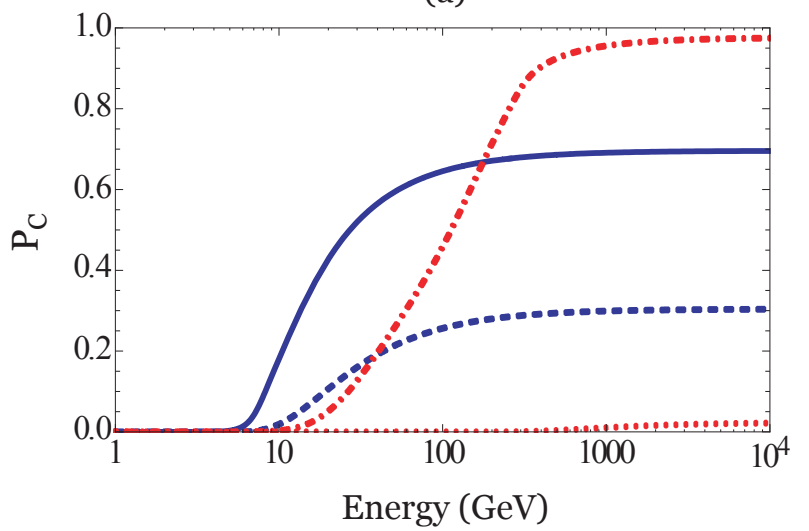

(b)

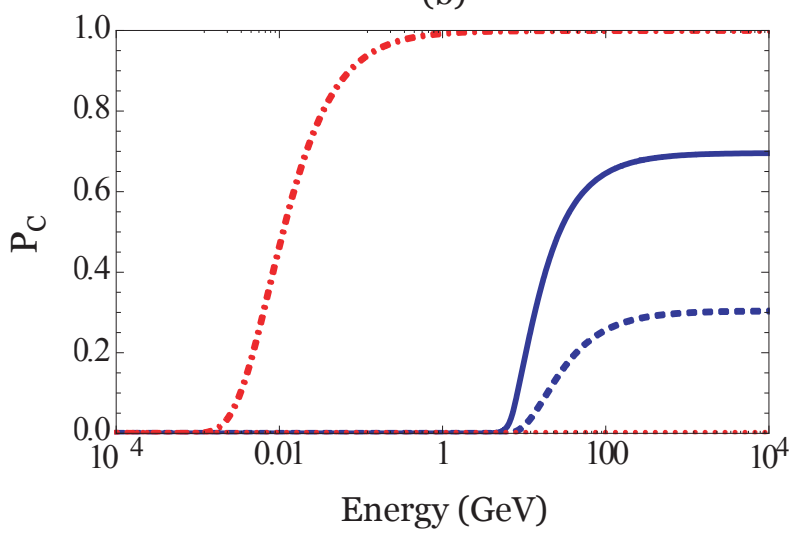

Figure 3. The level-crossing probabilities $P_{C}$; our chosen value of $\theta_{13}=8.8^{\circ}$ is used in fig (a), while fig (b) shows the corresponding result for $\theta_{13}=0.1^{\circ}$. The first $\left(\theta_{13}\right.$-driven) level crossing probabilities $\left(P_{C}^{h}\right)$ are shown by the red dot-dashed (neutrino) and dotted (antineutrino) lines, while the second $\left(\theta_{12}\right.$-driven) crossing probabilities $\left(P_{C}^{l}\right)$ are shown by the blue bold (neutrino) and dashed (antineutrino) lines.

neutrino energy) the lower level crossing takes place in the convective zone of the Sun where the solar density profile deviates from the exponential form assumed in eq. (3.7). We account for this by following the procedure developed in ref. [56], where $P_{C}$ is calculated according to eq. (3.7), but with $r_{0}$ replaced by an energy-dependent numerical fit.

The level crossing probabilities, for neutrinos and antineutrinos, are plotted as a function of neutrino energy in figure 3. Figure 3(a) shows the probabilities used here, assuming $\theta_{13}=8.8^{\circ}$, as suggested by recent T2K [58] and the Daya Bay [59] results, while figure $3(\mathrm{~b})$ shows the probabilities if we instead allow $\theta_{13}=0.1^{\circ}$. Note that for very small $\theta_{13}, P_{C}^{h}$ for neutrinos approaches 1 for all energies (i.e. the resonance is completely non-adiabatic). This is to be expected because as $\theta_{13} \rightarrow 0$ the heaviest vacuum eigenstate becomes completely decoupled, and does not mix with the other mass eigenstates. For antineutrinos $P_{C}^{h} \simeq 0$, as the resonance condition is never met. We also see that the lower crossing probabilities, $P_{C}^{l}$ are comparatively larger for neutrinos than antineutrinos, as $\bar{\nu}_{e}$ is produced in the lightest matter mass eigenstate and, again, does not undergo a resonance.

We apply eq. (3.9) to obtain the neutrino fluxes at the edge of the Sun, in the vacuum mass basis. The neutrinos propagate from the Sun to the Earth in these vacuum states, and can then be converted back to the flavour basis. The final spectra are shown in figure 4 .

\subsection{Final spectra}

The final neutrino spectra, per annihilation, for the electroweak bremsstrahlung annihilation mode are shown in figure 4, for various choices of the branching ratios to the lepton flavours. Also shown, for comparison, are the spectra that would result from DM annihiation to $W^{+} W^{-}$and $\tau^{+} \tau^{-}$final states. These comparison spectra were calculated using the same procedure described in section 2 .

We note that while the initial spectra at production were sensitive to flavour branching ratios chosen, neutrino flavour mixing in the Sun considerably reduces these differences. For example, for branching only to the electron flavour, the only $\nu_{\mu}$ and $\nu_{\tau}$ components at production are secondary neutrinos. However, the flavour mixing that takes places via the level crossings serves to even out the distribution across the three flavours. The final spectra 

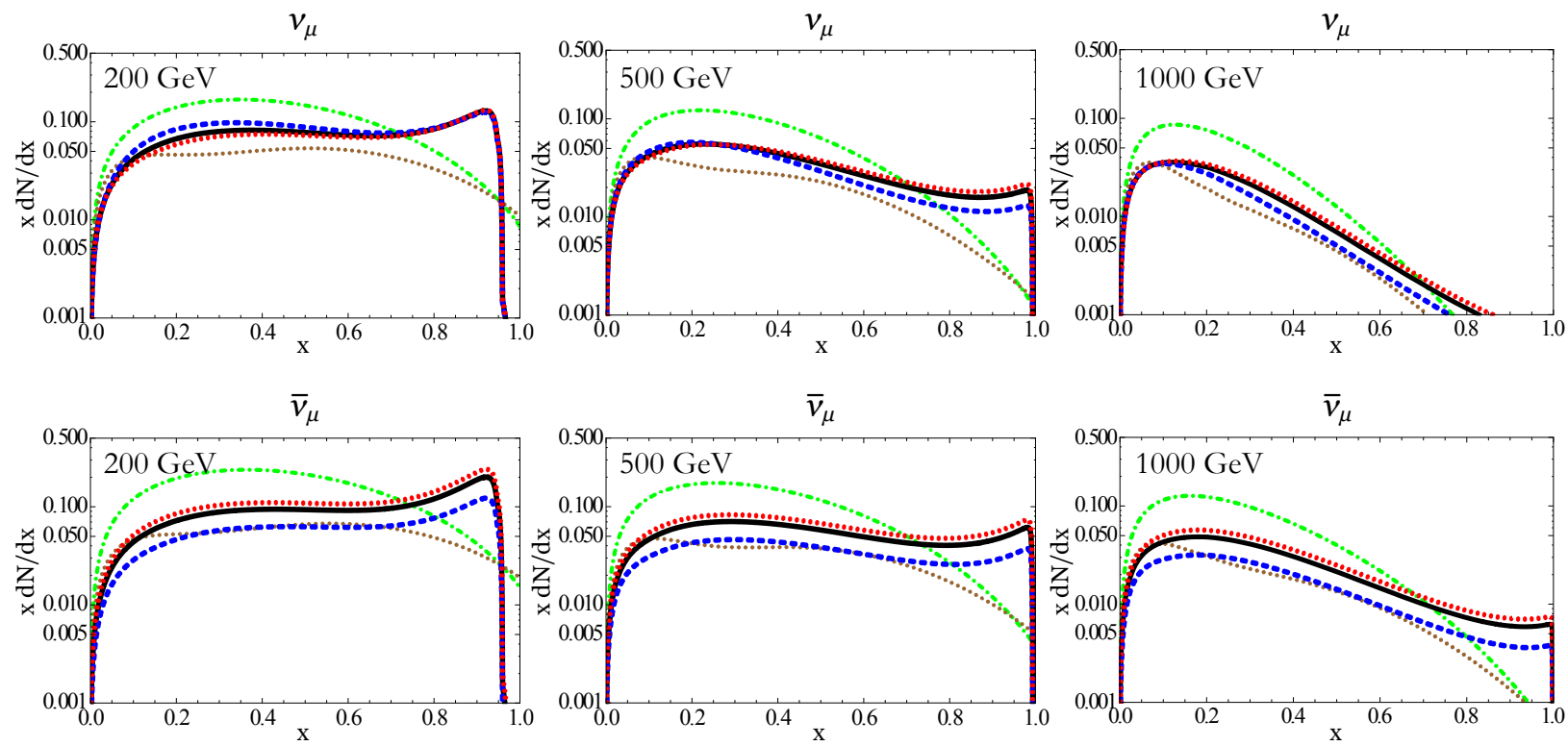

Figure 4. The final muon-flavour (anti)neutrino energy spectra, per annihilation, after propagation from the Sun's centre to Earth, for $M_{\chi}=200,500,1000 \mathrm{GeV}$. The curves denote different annihilation branching ratios: equal branching to all lepton flavours (black, bold), branching only to electron flavour (blue, dashed), branching evenly to $\mu$ and $\tau$ flavours (red, bold dotted). Also shown for comparison are the spectra arising from DM annihilation directly to $\tau^{+} \tau^{-}$(green, dot-dashed) and to $W^{+} W^{-}$(brown, dotted). We set $\theta_{13} \simeq 8.8^{\circ}$, and $\mu=1.2$.

have a similar shape, irrespective of the branching ratios chosen, with magnitudes that vary by only a factor of $\lesssim 2$.

Scattering and absorption have a strong effect on the spectra for $M_{\chi}=1000 \mathrm{GeV}$, but very little effect for $M_{\chi}=200 \mathrm{GeV}$. This is expected as absorption depends exponentially on the neutrino energy. For large $M_{\chi}$, the high energy regions of the spectra are strongly damped, removing the high energy peak. This is a bigger effect for neutrinos than for antineutrinos due to the different values of $\mathcal{E}$ - the scattering in the Sun affects neutrinos more strongly than their antiparticles. Overall, absorption significantly reduces the number of detectable neutrinos produced per annihilation. For the energies we consider, regeneration, which reintroduces neutrinos creating a pile-up at lower energies, is a smaller effect.

These plots in figure 4 were produced assuming a value for $\theta_{13}$ of $8.8^{\circ}$. For the purposes of comparison, we also computed spectra for $\theta_{13}=0.1^{\circ}$. In the $\theta_{13} \simeq 0$ limit, the evolution through the high energy $\theta_{13}$-driven resonance is completely non-adiabatic, with $P_{C}^{h} \simeq 1$ for all energies of interest, while for the larger mixing angle the resonance is (partially) adiabatic for energies $\lesssim 1000 \mathrm{GeV}$. In figure 5 , we compare the neutrino spectra for the two choices of $\theta_{13}$. Decreasing $\theta_{13}$ decreases the adiabaticity of the resonance, and increases the differences between the spectra for each flavour. This effect is most significant at low energies, where the level-crossing probabilities are small (see figure 3), while at high energies $P_{C}^{h} \rightarrow 1$ irrespective of the value of $\theta_{13}$. For antineutrinos, there is very little sensitivity to $\theta_{13}$ as the $\theta_{13}$-resonance condition is never met. From here on, we shall assume equal branching to all three flavours $\left(f_{e}=f_{\mu}=f_{\tau}\right)$, which renders the results insensitive to the value of $\theta_{13}$. 


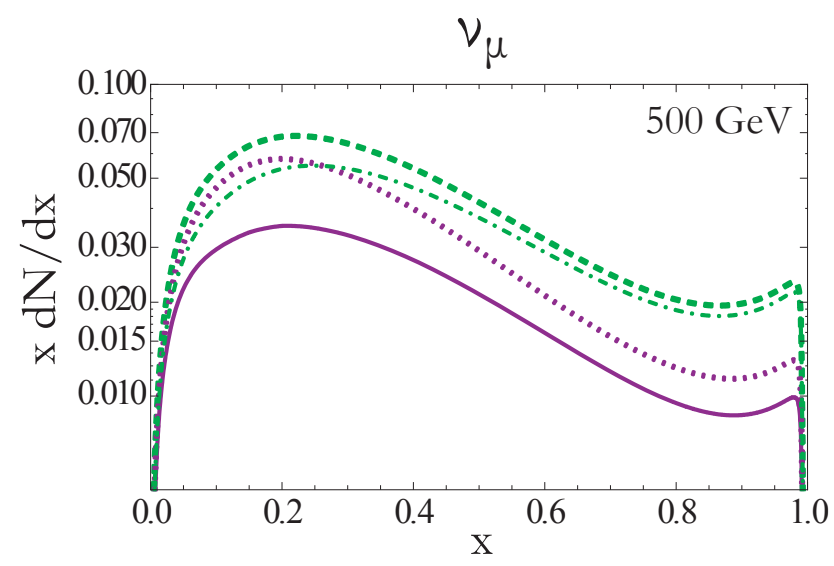

Figure 5. The $\nu_{\mu}$ spectra for $M_{\chi}=500 \mathrm{GeV}$, for different choices of of $\theta_{13}$, and where $\mu=1.2$. For branching only to $e$-flavoured leptons the spectra are shown for $\theta_{13}=0.1^{\circ}$ (purple, solid) and $\theta_{13}=8.8^{\circ}$ (purple, dotted). For branching to $\mu$ and $\tau$ flavours equally the curves correspond to $\theta_{13}=0.1^{\circ}$ (green, dashed) and $\theta_{13}=8.8^{\circ}$ (green, dot-dashed). Note that there is no significant dependence on $\theta_{13}$ for the case of equal branching to all flavours.

\section{Signals at Earth}

We now compare the signal from EW bremsstrahlung annihilation, to that arising in models in which DM annihilates instead to 2-body final states such as $W^{+} W^{-}$or $\tau^{+} \tau^{-}$. These channels have been chosen for comparison as they both have relatively hard neutrino spectra, with a significant flux at large values of $x$. As a result the existing limits on these channels [16-18] are more stringent than those with softer neutrino spectra. As seen in figure 4, the EW bremsstrahlung, $W^{+} W^{-}$and $\tau^{+} \tau^{-}$channels all have broad energy spectra, of similar shape and scale. However the EW bremsstrahlung channel has a significant high energy peak at $x \sim 1$, which will improve its detectability compared to the $W^{+} W^{-}$mode for which limits are commonly determined. We estimate relative event rates in the following subsection.

The spectra in figure 4 have been computed per annihlation; the absolute normalisation of the flux is specified by eq. 2.1. (If we assume capture-annihilation equilibrium, the absolute normalization of the event rate is set by the capture rate alone times the annihilation branching ratio of the channel of interest.) However, for a given annihilation rate (which we leave as a free parameter) it is useful to compare the ratio of event rates for possible annihilation channels. We can thus assess the detectability of the EW bremsstrahlung annihilation channel relative to that for the comparison 2-body final state channels, $W^{+} W^{-}$and $\tau^{+} \tau^{-}$, by comparing their relative event rates.

\subsection{Muon event rates}

We consider muon event rates in a detector such as IceCube. Although IceCube is able to detect neutrinos of all flavours via cascade events, we consider here only the muon-track events as they have the greatest detection probability, since the long muon range in ice allows the detection of muons created well outside the detector.

We estimate event rates following a similar procedure to that in refs. [55, 67-69]. A muon neutrino can undergo charged current scattering with a nucleon within the ice of the detector, to produce a muon with energy $E_{\mu}=E_{\nu}(1-y)$, where $y$ is the charged current inelasticity parameter. This muon will then propagate through the ice and lose energy, with 
a range given by

$$
R_{\mu}\left(E_{\mu}, E_{\mu}^{\mathrm{thr}}\right)=\frac{1}{\beta} \ln \left[\frac{\alpha+\beta E_{\mu}}{\alpha+\beta E_{\mu}^{\mathrm{thr}}}\right],
$$

where $\alpha=2.0 \mathrm{MeV} \mathrm{cm} / \mathrm{g}, \beta=4.2 \times 10^{-6} \mathrm{~cm}^{2} / \mathrm{g}$, and $E_{\mu}^{\text {thr }}$ is the muon energy detection threshold, taken to be $100 \mathrm{GeV}$. Therefore, the probability that a neutrino with energy $E_{\nu}$ creates a muon that is detected with an energy above $E_{\mu}^{\text {thr }}$ is given by

$$
P\left(E_{\nu}, E_{\mu}^{\mathrm{thr}}\right)=\rho_{N} N_{A} \sigma_{\text {nucleon }}\left(E_{\nu}\right) R_{\mu}\left(E_{\nu}(1-y), E_{\mu}^{\mathrm{thr}}\right),
$$

where $\rho_{N}$ is the target nucleon density, $N_{A}$ is Avogadro's number, and $\sigma_{\text {nucleon }}\left(E_{\nu}\right)$ is the charged current scattering cross section in ice. Finally, the muon track event rate is proportional to

$$
\int \frac{d N\left(E_{\nu}\right)}{d E_{\nu}} P\left(E_{\nu}, E_{\mu}^{\mathrm{thr}}\right) A^{\mathrm{eff}} d E_{\nu}
$$

where $A^{\text {eff }}$ is the effective area of the detector; for IceCube this is $\sim 1 \mathrm{~km}^{2}$ [70]. The scattering cross section $\sigma_{\text {nucleon }}\left(E_{\nu}\right)$ is proportional to $E_{\nu}$ and, in ice, the neutrino cross section is approximately twice as large as that for antineutrinos. We choose the average values of $y$ as tabulated in ref. [67], which are approximately $\sim 0.45$ and $\sim 0.35$ for neutrinos and antineutrinos respectively. As a result, the lower bound on the (anti)neutrino energies that will produce observable muons is

$$
\begin{array}{rlrl}
E_{\nu_{\mu}} & \geq E_{\mu}^{\mathrm{thr}} /\left(1-y_{\nu}\right), & E_{\bar{\nu}_{\mu}} & \geq E_{\mu}^{\mathrm{thr}} /\left(1-y_{\bar{\nu}}\right), \\
& \simeq 182 \mathrm{GeV}, & \simeq 154 \mathrm{GeV} .
\end{array}
$$

In table 4.1, we show the ratio of muon-track events for various annihilation modes, assuming the same overall normalisation. For example, by setting the branching ratio in the table equal to 1 , we can compare the event rate for a model in which the annihilation proceeds via bremsstrahlung with that for a model in which the annihilation is solely to $W^{+} W^{-}$or $\tau^{+} \tau^{-}$, assuming the same total annihilation rate.

We see that, for a given annihilation rate, annihilation via the EW bremsstrahlung channel would produce more events than annihilation via a $W^{+} W^{-}$channel, for all masses considered, with a ratio of $\sim 4$ at $M_{\chi}=200 \mathrm{GeV}$. The ratio is largest for low dark matter masses, because only the high energy regions of the spectra are above the detection threshold, and hence the high energy peak in the EW bremsstrahlung spectrum dominates. The ratio is smaller for large $M_{\chi}$, as the detection threshold then corresponds to smaller values of $x$, and absorption effects damp the spectra at high energy. There is only a mild dependence of these results on the value of the mass degeneracy, $\mu=M_{\eta}^{2} / M_{\chi}^{2}$. For larger values of $\mu$ the high energy peak is diminished, but more mid-to-high energy neutrinos are produced, such that the total number of detectable events remains approximately constant.

Detection prospects for annihilation channels which produce hard spectra, such as $W^{+} W^{-}$, are much better than those for annihilation channels which produce much softer neutrino spectra, such as $\bar{q} q$. Given that annihilation via the bremsstrahlung channel would result in more events than annihilation to $W^{+} W^{-}$, its detection prospects appear promising. The experimental sensitivity for a given annihilation channel is usually expressed in terms of a limit on the DM-nucleon scattering cross section which controls the absolute event rate (assuming capture-annihilation equilibrium). For annihilation to purely $W^{+} W^{-}$, the projected IceCube sensitivity (180 days of the full 86 string configuration) to spin-dependent 


\begin{tabular}{|c||c|c||c|c||c|c||}
\hline \multicolumn{1}{|c||}{} & \multicolumn{2}{c||}{$200 \mathrm{GeV}$} & \multicolumn{2}{c||}{$500 \mathrm{GeV}$} & \multicolumn{2}{c||}{$1000 \mathrm{GeV}$} \\
& $\mu=1.2$ & $\mu=5$ & $\mu=1.2$ & $\mu=5$ & $\mu=1.2$ & $\mu=5$ \\
\hline$\frac{\Phi_{\nu}(\text { brem })}{\Phi_{\nu}\left(\tau^{+} \tau^{-}\right)} / \frac{\operatorname{Br}(\mathrm{brem})}{\operatorname{Br}\left(\tau^{+} \tau^{-}\right)}$ & 1.8 & 2.1 & 1.2 & 1.2 & 0.6 & 0.7 \\
\hline$\frac{\Phi_{\nu}(\mathrm{brem})}{\Phi_{\nu}\left(W^{+} W^{-}\right)} / \frac{\mathrm{Br}(\mathrm{brem})}{\operatorname{Br}\left(W^{+} W^{-}\right)}$ & 3.8 & 4.6 & 2.4 & 2.6 & 1.9 & 2.0 \\
\hline
\end{tabular}

Table 1. The ratio of $\mu^{ \pm}$muon-track events in an IceCube-like detector, for various annihilation channels. We have assumed the same overall normalization for each possible annihilation mode, that is, assuming capture-annihilation equilibrium to hold, we assume the same capture rate for each case. For the EW bremsstrahlung annihilation channel, equal branching ratios to all lepton flavours was assumed $\left(f_{e}=f_{\mu}=f_{\tau}\right)$.

DM-nucelon scattering is $\sigma_{\chi N}^{S D} \sim$ few $\times 10^{-5} \mathrm{pb}$ [71] for the DM mass range we consider. Since annihilation purely via the bremmstrahlung channel will produce more events than the $W^{+} W^{-}$channel by a factor of a few, we estimate a sensitivity of $\sigma_{\chi N}^{S D} \sim 1 \times 10^{-5}$ pb for this channel (see also ref. [72]).

\subsection{Parameter constraints}

We have presented spectra per annihilation, in the spirit of ref. [9], choosing to leave the absolute normalization as a free parameter. However, the detection prospects for a given DM model depend on the absolute event rate, which is determined by eq. 2.1 and eq. 2.2 and, in general, will depend on both the DM-nucleon scattering and the DM annihilation cross sections. However, importantly, if capture-annihilation equilibrium is established, then the event rate is controlled by the DM-nucleon scattering cross section alone (modulo astrophysical uncertainties in the local dark matter density and velocity). We now discuss whether this condition is expected to hold in the DM models of interest for electroweak bremsstrahlung.

Expressions for the capture and annihilation rate are given, e.g., in ref. [46]. In order for equilibrium to be established, the product of (i) the DM-nucleon scattering and (ii) the DM annihilation cross section must be sufficiently large. If one assumes a spin dependent DM-nucleon scattering cross section of $\sim 10^{-5} \mathrm{pb}$ (the IceCube sensitivity estimated above), an annihilation cross section of $\langle\sigma v\rangle \gtrsim 10^{-2}\langle\sigma v\rangle_{\text {th }}$ is required for capture-annihilation equilibrium to be reached within the lifetime of the solar system, where $\langle\sigma v\rangle_{\text {th }} \simeq 2 \times 10^{-26} \mathrm{~cm}^{3} / \mathrm{s}$ is the annihilation cross section expected for a thermal relic [73]. We discuss each of these cross sections in turn.

The bremsstrahlung annihilation cross section is expected to be approximately two orders of magnitude smaller than that for a thermal relic, $\langle\sigma v\rangle_{\mathrm{brem}} \sim 10^{-2}\langle\sigma v\rangle_{\mathrm{th}}{ }^{2}$ This is because the annihilation cross section at thermal freezeout is dominated not by the bremsstrahlung channels, but instead by annihilation to 2-body final states. The 3-body bremsstrahlung processes dominate the annihilation in the late universe, because the 2-body

\footnotetext{
${ }^{2}$ Larger annihilation cross sections would imply either a subdominant DM component, or that the DM was produced not by thermal freezeout but by some more exotic mechanism. A number of recent papers have considered indirect detection and set upper limits on the EW bremsstrahlung annihilation cross section based on observations of antiprotons and gamma rays. For instance refs. [30, 39] both find limits of approximately $\langle\sigma v\rangle \lesssim 10^{-24}-10^{-25} \mathrm{~cm}^{3} / \mathrm{s}$ over the mass range $100-300 \mathrm{GeV}$. Ref. [42] performs a search for a bremsstrahlung annihilation signal in the Fermi LAT gamma ray data and sets bounds of approximately $\langle\sigma v\rangle \lesssim 10^{-27} \mathrm{~cm}^{3} / \mathrm{s}$ over the same mass range.
} 
final states are p-wave suppressed by a factor $v^{2} \sim 10^{-6}$; in the early universe $v$ is much larger and the 2-body final state is not strongly suppressed. See the discussion in section 4 of ref [42] for further details.

For a purely leptophilic model, the DM couplings to quarks arise at loop level. For the coupling of eq. 2.3 the DM-nucleon interaction arises at 1-loop level through a coupling to the $\mathrm{Z}$ boson, resulting in an effective interaction ([48])

$$
\mathcal{L}_{A}=\frac{\mathcal{G}}{M_{Z}^{2}}\left(\bar{\chi} \gamma^{\mu} \gamma_{5} \chi\right)\left(\bar{q} \gamma_{\mu} \gamma_{5} q\right),
$$

where $\mathcal{G}$ is the loop-induced form factor. The resulting spin-dependent DM-nucleon cross section is shown in figure 2 (a) of ref.[48], and can take values $\lesssim 10^{-6} \mathrm{pb}$.

Therefore, for the purely leptophilic model described by the coupling of eq. 2.3 , the product of the DM-nucleon scattering and DM annihilation cross sections are sufficiently small that capture-annihilation equilibrium would not hold (although we would be close to this limit, with event rates suppressed by a factor of less than 10 for some parameters). However, if the model were not purely leptophilic, the DM-nucleon cross section could easily be larger, such that capture-annihilation equilibrium would hold. For instance, couplings identical to that we consider arise in SUSY models. Embedding the toy model of eq. 2.3 into a full theory (e.g. the MSSM, or other theory beyond the standard model) is a more realistic scenario. In that case it is likely that several annihilation channels would be open, which would need to be combined with model dependent branching ratios. In this paper we take a purely phenomenological approach to study the EW bremsstrahlung signal in isolation.

\section{Conclusions}

High-energy neutrinos produced by the annihilation of dark matter captured in the Sun offer a useful dark matter search strategy. By comparing the predicted neutrino fluxes with future detections, we can potentially distinguish between different DM annihilation channels, thus providing information to discriminate among competing DM models.

DM annihilation is usually assumed to be dominated by annihilation to 2-body final states. However, there are important DM annihilation processes with 3-body final states, namely bremsstrahlung processes in which a $W, Z$ or $\gamma$ is radiated. The electroweak bremsstrahlung processes $\chi \chi \rightarrow \bar{f} f Z$ and $\chi \chi \rightarrow f \nu W$ have been shown to be the dominant DM annihilation mode in certain popular models in which the lowest order annihilation processes are helicity suppressed. In the context of solar WIMP annihlation, these electroweak bremsstrahlung annihilation channels produce large, energetic, fluxes of neutrinos.

We have computed the neutrino energy spectra resulting from DM annihilation via the electroweak bremsstrahlung channel. This consists of primary neutrinos produced directly in the annihilations, which have a distinguishing peak near the endpoint $E_{\nu}=M_{\chi}$, and secondary neutrinos produced in decays of annihilation products. By comparing the event rates for the electroweak bremsstrahlung annihilation channel with those for annihilation instead to $W^{+} W^{-}$, we have shown that the EW bremsstrahlung channel has the larger event rate per annihilation and hence promising detection prospects.

\section{Acknowledgments}

AJB was supported by the Commonwealth of Australia and NFB by the Australian Research Council. We thank Thomas Weiler, Kalliopi Petraki, James Dent, Lawrence Krauss, Nicholas 
Setzer and Martin White for helpful discussions.

\section{References}

[1] J. Silk, K. Olive, and M. Srednicki, The photino, the sun, and high-energy neutrinos, Phys.Rev.Lett. 55 (1985) 257-259.

[2] W. H. Press and D. N. Spergel, Capture by the Sun of a Galactic Population of Weakly Interacting Massive Particles, Astrophys.J. 296 (1985) 679-684.

[3] K. Freese, Can scalar neutrinos or massive Dirac neutrinos be the missing mass?, Phys.Lett. B167 (1986) 295-300.

[4] L. Krauss, M. Srednicki, and F. Wilczek, Solar system constraints and signatures for dark-matter candidates, Phys.Rev. D33 (1986) 2079.

[5] T. Gaisser, G. Steigman, and S. Tilav, Limits on cold-dark-matter candidates from deep underground detectors, Phys.Rev. D34 (1986) 2206.

[6] A. Gould, Resonant Enhancements in WIMP Capture by the Earth, Astrophys.J. 321 (1987) 571.

[7] M. Srednicki, K. Olive, and J. Silk, High-energy neutrinos from the sun and cold dark matter, Nucl.Phys. B279 (1987) 804-823.

[8] M. Kamionkowski, Energetic neutrinos from heavy-neutralino annihilation in the sun, Phys.Rev. D44 (1991) 3021-3042.

[9] M. Cirelli, N. Fornengo, T. Montaruli, I. A. Sokalski, A. Strumia, et al., Spectra of Neutrinos from Dark Matter Annihilations, Nucl.Phys. B727 (2005) 99-138, [hep-ph/0506298].

[10] M. Blennow, J. Edsjö, and T. Ohlsson, Neutrinos from WIMP annihilations obtained using a full three-flavor Monte Carlo approach, JCAP 01 (2008) 021, [arXiv:0709.3898].

[11] V. Barger, F. Halzen, D. Hooper, and C. Kao, Indirect search for neutralino dark matter with high energy neutrinos, Phys.Rev. D65 (2002) 075022, [hep-ph/0105182v1].

[12] R. Lehnert and T. J. Weiler, Flavor Sensitivity to $\theta_{13}$ and the Mass Hierarchy for neutrinos from Solar WIMP Annihilation, arXiv:1002.2441.

[13] A. Erkoca, M. Reno, and I. Sarcevic, Muon fluxes from dark matter annihilation, Phys.Rev. D80 (2009) 043514, [arXiv:0906.4364].

[14] K. Belotsky, M. Khlopov, and C. Kouvaris, Muon flux limits for Majorana dark matter from strong coupling theories, Phys. Rev. D79 (2009) 083520, [arXiv:0810.2022].

[15] V. Barger, J. Kumar, D. Marfatia, and E. M. Sessolo, Fermion WIMPless Dark Matter at DeepCore and IceCube, Phys.Rev. D81 (2010) 115010, [arXiv:1004.4573].

[16] Super-Kamiokande Collaboration, K. Abe et al., An Indirect Search for WIMPs in the Sun using 3109.6 days of upward-going muons in Super-Kamiokande, astro-ph/1108.3384.

[17] IceCube Collaboration, R. Abbasi et al., Limits on a muon flux from Kaluza-Klein dark matter annihilations in the Sun from the IceCube 22-string detector, Phys.Rev. D81 (2010) 057101, [astro-ph/0910.4480v1].

[18] IceCube Collaboration, R. Abbasi et al., Limits on a muon flux from neutralino annihilations in the Sun with the IceCube 22-string detector, Phys.Rev.Lett. 102 (2009) 201302, [astro-ph/0902.2460v3].

[19] IceCube Collaboration, T. DeYoung, Particle physics in ice with IceCube DeepCore, Nucl.Instrum.Meth. A (2011) [arXiv:1112.1053]. 
[20] N. Bell, J. Dent, A. Galea, T. Jacques, L. Krauss, and T. Weiler, W/Z Bremsstrahlung as the Dominant Annihilation Channel for Dark Matter, Revisited, Phys.Lett. B706 (2011) [arXiv: 1104.3823].

[21] N. F. Bell, J. B. Dent, T. D. Jacques, and T. J. Weiler, W/Z Bremsstrahlung as the Dominant Annihilation Channel for Dark Matter, Phys.Rev. D83 (2011) 013001, [arXiv: 1009.2584].

[22] L. Bergström, Radiative Processes in Dark Matter Photino Annihilation, Phys.Lett. B225 (1989) 372.

[23] R. Flores, K. Olive, and S. Rudaz, Radiative processes in LSP annihilation, Phys.Lett. B232 (1989) 377-382.

[24] E. Baltz and L. Bergström, Detection of Leptonic Dark Matter, Phys.Rev. D67 (2003) 043516, [hep-ph/0211325].

[25] T. Bringmann, L. Bergström, and J. Edsjö, New Gamma-Ray Contributions to Supersymmetric Dark Matter Annihilation, JHEP 0801 (2008) 049, [hep-ph/0710.3169v3].

[26] L. Bergström, T. Bringmann, and J. Edsjö, New Positron Spectral Features from Supersymmetric Dark Matter: A Way to Explain the PAMELA Data?, Phys.Rev. D78 (2008) 103520, [astro-ph/0808.3725v3].

[27] V. Barger, Y. Gao, W. Keung, and D. Marfatia, Generic dark matter signature for gamma-ray telescopes, Phys. Rev. D 80 (Sep, 2009) 063537, [hep-ph/0906.3009v2].

[28] H. Goldberg, Constraint on the photino mass from cosmology, Phys.Rev.Lett. 50 (1983) $1419-1422$.

[29] L. Krauss, New constraints on "INO" masses from cosmology, Nucl.Phys. B227 (1983) $556-569$.

[30] N. F. Bell, J. B. Dent, T. D. Jacques, and T. J. Weiler, Dark Matter Annihilation Signatures from Electroweak Bremsstrahlung, Phys.Rev. D84 (2011) 103517, [arXiv:1101.3357].

[31] N. F. Bell, J. B. Dent, T. D. Jacques, and T. J. Weiler, Electroweak Bremsstrahlung in Dark Matter Annihilation, Phys.Rev. D78 (2008) 083540, [arXiv:0805.3423].

[32] V. Barger, W. Keung, and D. Marfatia, Bremsstrahlung in dark matter annihilation, Phys.Lett. B707 (2012) [arXiv:1111.4523].

[33] P. Ciafaloni and D. Comelli, Sudakov Effects in Electroweak Corrections, Phys.Lett. B446 (1999) 278-284, [hep-ph/9809321].

[34] P. Ciafaloni and A. Urbano, TeV scale Dark Matter and Electroweak Radiative Corrections, Phys.Rev. D82 (2010) 043512, [arXiv:1001.3950].

[35] P. Ciafaloni, D. Comelli, A. Riotto, F. Sala, A. Strumia, and A. Urbano, Weak corrections are relevant for dark matter indirect detection, JCAP 03 (2011) 019, [arXiv: 1009.0224].

[36] P. Ciafaloni, M. Cirelli, D. Comelli, A. De Simone, A. Riotto, and A. Urbano, On the importance of electroweak corrections for Majorana dark matter indirect detection, JCAP 06 (2011) 018, [arXiv:1104.2996].

[37] P. Ciafaloni, M. Cirelli, D. Comelli, A. De Simone, A. Riotto, and A. Urbano, Initial state radiation in Majorana Dark Matter annihilations, JCAP (2011) 034, [arXiv:1107.4453].

[38] M. Garny, A. Ibarra, and S. Vogl, Antiproton constraints on dark matter annihilations from internal electroweak bremsstrahlung, JCAP 07 (2011) 028, [arXiv:1105.5367].

[39] M. Garny, A. Ibarra, and S. Vogl, Dark matter annihilations into two light fermions and one gauge boson: general analysis and antiproton constraints, arXiv:1112.5155.

[40] P. Ciafaloni, D. Comelli, A. De Simone, A. Riotto, and A. Urbano, Electroweak bremsstrahlung for wino-like Dark Matter annihilations, arXiv:1202.0692. 
[41] M. Kachelriess, P. Serpico, and M. Solberg, Role of electroweak bremsstrahlung for indirect dark matter signatures, Phys. Rev. D80 (2009) 123533, [arXiv:0911.0001].

[42] T. Bringmann, X. Huang, A. Ibarra, S. Vogl, and C. Weniger, Fermi LAT Search for Internal Bremsstrahlung Signatures from Dark Matter Annihilation, arXiv:1203.1312.

[43] K. Griest and D. Seckel, Cosmic Asymmetry, Neutrinos and the Sun, Nucl.Phys. B283 (1987) 681.

[44] A. Bottino, G. Fiorentini, N. Fornengo, B. Ricci, S. Scopel, and F. Villante, Does solar physics provide constraints to weakly interacting massive particles?, Phys.Rev. D66 (2002) 053005, [hep-ph/0206211].

[45] J. Lundberg and J. Edsjö, Weakly interacting massive particle diffusion in the solar system including solar depletion and its effect on Earth capture rates, Phys.Rev. D69 (2004) 123505, [astro-ph/0401113].

[46] G. Bertone, D. Hooper, and J. Silk, Particle Dark Matter: Evidence, Candidates and Constraints, Phys.Rept. 405 (2005) 279-390, [hep-ph/0404175].

[47] G. Jungman, M. Kamionkowski, and K. Griest, Supersymmetric Dark Matter, Phys.Rept. 267 (1996) 195-373, [hep-ph/9506380].

[48] Q.-H. Cao, E. Ma, and G. Shaughnessy, Dark Matter: The Leptonic Connection, Phys.Lett. B673 (2009) 152-155, [arXiv:0901.1334].

[49] PAMELA Collaboration, O. Adriani et al., An Anomalous Positron Abundance in Cosmic Rays with Energies 1.5-100 GeV, Nature 458 (2009) 607-609, [arXiv:0810.4995].

[50] PAMELA Collaboration, O. Adriani et al., PAMELA Results on the Cosmic-Ray Antiproton Flux from $60 \mathrm{MeV}$ to $180 \mathrm{GeV}$ in Kinetic Energy, Phys.Rev.Lett. 105 (2010) 121101, [arXiv: 1007.0821].

[51] PAMELA Collaboration, O. Adriani et al., A New Measurement of the Antiproton-to-Proton Flux Ratio up to 100 GeV in the Cosmic Radiation, Phys.Rev.Lett. 102 (2009) 051101, [arXiv:0810.4994].

[52] F. Halzen and D. Hooper, The Indirect Search for Dark Matter with IceCube, New J.Phys. 11 (2009) 105019, [arXiv:0910.4513].

[53] IceCube Collaboration, R. Abbasi et al., The design and performance of IceCube DeepCore, Astropart.Phys. 35 (2012) [arXiv:1109.6096].

[54] T. Sjöstrand, S. Mrenna, and P. Z. Skands, A Brief Introduction to PYTHIA 8.1, Comput.Phys.Commun. 178 (2008) 852-867, [arXiv:0710.3820].

[55] N. Bell and K. Petraki, Enhanced Neutrino Signals from Dark Matter Annihilation in the Sun via Metastable Mediators, JCAP 04 (2011) 003, [arXiv:1102.2958].

[56] A. Friedland, On the Evolution of the Neutrino State Inside the Sun, Phys.Rev. D64 (2001) 013008, [hep-ph/0010231].

[57] Particle Data Group Collaboration, K. Nakamura et al., Review of Particle Physics, J.Phys. G37 (2010) 075021.

[58] T2K Collaboration, K. Abe et al., Indication of Electron Neutrino Appearance from an Accelerator-produced Off-axis Muon Neutrino Beam, Phys.Rev.Lett. 107 (2011) 041801, [arXiv:1106.2822].

[59] Daya Bay Collaboration, F. An et al., Observation of electron-antineutrino disappearance at Daya Bay, arXiv:1203.1669.

[60] L. Wolfenstein, Neutrino Oscillations in Matter, Phys.Rev. D17 (1978) 2369-2374.

[61] S. Mikheev and A. Smirnov, Resonance enhancement of oscillations in matter and solar 
neutrino spectroscopy, Sov. J. Nucl. Phys.(Engl. Transl.) 42 (1985).

[62] S. Toshev, Exact Analytical Solution of the Two Neutrino Evolution Equation in Matter with Exponentially Varying Density, Phys.Lett. B196 (1987) 170.

[63] A. Strumia and F. Vissani, Neutrino masses and mixings and..., hep-ph/0606054.

[64] R. Lehnert and T. J. Weiler, Neutrino Flavor Ratios as Diagnostic of Solar WIMP Annihilation, Phys.Rev. D77 (2008) 125004, [arXiv:0708.1035].

[65] S. Mikheev and A. Smirnov, Resonance oscillations of neutrinos in matter, Sov.Phys.Usp. 30 (1987) 759 .

[66] M. Kachelriess and R. Tomas, Nonadiabatic level crossing in resonant and nonresonant neutrino oscillations, Phys.Rev. D64 (2001), no. 7073002.

[67] R. Gandhi, C. Quigg, M. H. Reno, and I. Sarcevic, Ultrahigh-Energy Neutrino Interactions, Astropart.Phys. 5 (1996) 81-110, [hep-ph/9512364].

[68] S. Dutta, M. Reno, I. Sarcevic, and D. Seckel, Propagation of Muons and Taus at High Energies, Phys.Rev. D63 (2001) 094020, [hep-ph/0012350].

[69] J. F. Beacom, N. F. Bell, D. Hooper, S. Pakvasa, and T. J. Weiler, Measuring Flavor Ratios of High-Energy Astrophysical Neutrinos, Phys.Rev. D68 (2003) 093005, [hep-ph/0307025].

[70] IceCube Collaboration, J. Ahrens et al., Sensitivity of the IceCube Detector to Astrophysical Sources of High Energy Muon Neutrinos, Astropart.Phys. 20 (2004) 507-532, [astro-ph/0305196].

[71] IceCube Collaboration, R. Abbasi et al., Multi-year search for dark matter annihilations in the Sun with the AMANDA-II and IceCube detectors, Phys. Rev. D85 (2012) 042002, [arXiv:1112.1840].

[72] K. Fukushima, Y. Gao, J. Kumar, and D. Marfatia, Bremsstrahlung signatures of dark matter annihilation in the Sun, arXiv:1208.1010.

[73] G. Steigman, B. Dasgupta, and J. F. Beacom, Precise relic WIMP abundance and its impact on searches for dark matter annihilation, Phys. Rev. D86 (2012) 023506, [arXiv:1204.3622]. 